\title{
Some properties of certain subclasses of analytic functions involving a differential operator
}

\section{Loriana Andrei*}

\section{"Correspondence:}

lori_andrei@yahoo.com Department of Mathematics and Computer Science, University of Oradea, 1 Universitatii street, Oradea, 410087, Romania

\begin{abstract}
In the present paper, we introduce and study certain subclasses of analytic functions in the open unit disk $U$ which is defined by the differential operator $D R_{\lambda}^{m, n}$. We study and investigate some inclusion properties of these classes. Furthermore, a generalized Bernardi-Libera-Livington integral operator is shown to be preserved for these classes. MSC: $30 C 45$

Keywords: analytic functions; differential operator; differential subordination; differential superordination
\end{abstract}

\section{Introduction}

Let $\mathcal{A}$ be a class of functions $f$ in the open unit disk $U=\{z \in \mathbb{C}:|z|<1\}$ normalized by $f(0)=f^{\prime}(0)-1=0$. Thus each $f \in \mathcal{A}$ has a Taylor series representation

$$
f(z)=z+\sum_{j=2}^{\infty} a_{j} z^{j}
$$

We denote by $\mathcal{S}(\xi)$ the well-known subclass of $\mathcal{A}$ consisting of all analytic functions which are, respectively, starlike of order $\xi[1,2]$

$$
\mathcal{S}(\xi)=\left\{f \in \mathcal{A}: \operatorname{Re}\left(\frac{z f^{\prime}(z)}{f(z)}\right)>\xi, z \in U\right\}, \quad 0 \leq \xi<1 .
$$

Let $\mathcal{R}$ be a class of all functions $\phi$ which are analytic and univalent in $U$ and for which $\phi(U)$ is convex with $\phi(0)=1$ and $\operatorname{Re} \phi(z)>0, z \in U$.

For two functions $f$ and $g$ analytic in $U$, we say that the function $f$ is subordinate to $g$ in $U$ and write $f(z) \prec g(z), z \in U$, if there exists a Schwarz function $w(z)$ which is analytic in $U$ with $w(0)=0$ and $|w(z)|<1$ such that $f(z)=g(w(z)), z \in U$.

Making use of the principle of subordination between analytic functions, denote by $\mathcal{S}(\xi, \phi)$ [3] a subclass of the class $\mathcal{A}$ for $0 \leq \xi<1$ and $\phi \in \mathcal{R}$ which are defined by

$$
\mathcal{S}(\xi, \phi)=\left\{f \in \mathcal{A}: \frac{1}{1-\xi}\left(\frac{z f^{\prime}(z)}{f(z)}-\zeta\right) \prec \phi(z), z \in U\right\} .
$$

(O\#CPRAndrei; licensee Springer. This is an Open Access article distributed under the terms of the Creative Commons Attribution License (http://creativecommons.org/licenses/by/2.0), which permits unrestricted use, distribution, and reproduction in any medium, provided the original work is properly cited. 
Let $f, g \in \mathcal{A}$, where $f$ and $g$ are defined by $f(z)=z+\sum_{j=2}^{\infty} a_{j} z^{j}$ and $g(z)=z+\sum_{j=2}^{\infty} b_{j} z^{j}$. Then the Hadamard product (or convolution) $f * g$ of the functions $f$ and $g$ is defined by

$$
(f * g)(z)=z+\sum_{j=2}^{\infty} a_{j} b_{j} z^{j}
$$

Definition 1.1 (Al-Oboudi [4]) For $f \in \mathcal{A}, \lambda \geq 0$ and $m \in \mathbb{N}$, the operator $D_{\lambda}^{m}$ is defined by $D_{\lambda}^{m}: \mathcal{A} \rightarrow \mathcal{A}$,

$$
\begin{aligned}
& D_{\lambda}^{0} f(z)=f(z), \\
& D_{\lambda}^{1} f(z)=(1-\lambda) f(z)+\lambda z f^{\prime}(z)=D_{\lambda} f(z), \\
& \ldots, \\
& D_{\lambda}^{m} f(z)=(1-\lambda) D_{\lambda}^{m-1} f(z)+\lambda z\left(D_{\lambda}^{m} f(z)\right)^{\prime}=D_{\lambda}\left(D_{\lambda}^{m-1} f(z)\right), \quad z \in U .
\end{aligned}
$$

Remark 1.1 If $f \in \mathcal{A}$ and $f(z)=z+\sum_{j=2}^{\infty} a_{j} z^{j}$, then $D_{\lambda}^{m} f(z)=z+\sum_{j=2}^{\infty}[1+(j-1) \lambda]^{m} a_{j} z^{j}$, $z \in U$.

Remark 1.2 For $\lambda=1$ in the above definition, we obtain the Sălăgean differential operator [5].

Definition 1.2 (Ruscheweyh [6]) For $f \in \mathcal{A}$ and $n \in \mathbb{N}$, the operator $R^{n}$ is defined by $R^{n}$ : $\mathcal{A} \rightarrow \mathcal{A}$,

$$
\begin{aligned}
& R^{0} f(z)=f(z), \\
& R^{1} f(z)=z f^{\prime}(z), \\
& \ldots, \\
& (n+1) R^{n+1} f(z)=z\left(R^{n} f(z)\right)^{\prime}+n R^{n} f(z), \quad z \in U .
\end{aligned}
$$

Remark 1.3 If $f \in \mathcal{A}, f(z)=z+\sum_{j=2}^{\infty} a_{j} z^{j}$, then $R^{n} f(z)=z+\sum_{j=2}^{\infty} \frac{(n+j-1) !}{n !(j-1) !} a_{j} z^{j}, z \in U$.

Definition 1.3 ([7]) Let $\lambda \geq 0$ and $n, m \in \mathbb{N}$. Denote by $D R_{\lambda}^{m, n}: \mathcal{A} \rightarrow \mathcal{A}$ the operator given by the Hadamard product of the generalized Sălăgean operator $D_{\lambda}^{m}$ and the Ruscheweyh operator $R^{n}$,

$$
D R_{\lambda}^{m, n} f(z)=\left(D_{\lambda}^{m} * R^{n}\right) f(z)
$$

for any $z \in U$ and each nonnegative integer $m, n$.

Remark1.4 If $f \in \mathcal{A}$ and $f(z)=z+\sum_{j=2}^{\infty} a_{j} z^{j}$, then $D R_{\lambda}^{m, n} f(z)=z+\sum_{j=2}^{\infty}[1+(j-1) \lambda]^{m} \frac{(n+j-1) !}{n !(j-1) !} \times$ $a_{j}^{2} z^{j}, z \in U$.

Remark 1.5 The operator $D R_{\lambda}^{m, n}$ was studied also in [8-10].

For $\lambda=1, m=n$, we obtain the Hadamard product $S R^{n}[11]$ of the Sălăgean operator $S^{n}$ and the Ruscheweyh derivative $R^{n}$, which was studied in $[12,13]$. 
For $m=n$, we obtain the Hadamard product $D R_{\lambda}^{n}[14]$ of the generalized Sălăgean operator $D_{\lambda}^{n}$ and the Ruscheweyh derivative $R^{n}$, which was studied in [15-20].

Using a simple computation, one obtains the next result.

Proposition $1.1([7])$ For $m, n \in \mathbb{N}$ and $\lambda \geq 0$, we have

$$
D R_{\lambda}^{m+1, n} f(z)=(1-\lambda) D R_{\lambda}^{m, n} f(z)+\lambda z\left(D R_{\lambda}^{m, n} f(z)\right)^{\prime}
$$

and

$$
z\left(D R_{\lambda}^{m, n} f(z)\right)^{\prime}=(n+1) D R_{\lambda}^{m, n+1} f(z)-n D R_{\lambda}^{m, n} f(z) .
$$

By using the operator $D R_{\lambda}^{m, n} f(z)$, we define the following subclasses of analytic functions for $0 \leq \zeta<1$ and $\phi \in \mathcal{R}$ :

$$
\begin{aligned}
& \mathcal{S}_{\lambda}^{m, n}(\xi)=\left\{f \in \mathcal{A}: D R_{\lambda}^{m, n} f \in \mathcal{S}(\xi)\right\}, \\
& \mathcal{S}_{\lambda}^{m, n}(\xi, \phi)=\left\{f \in \mathcal{A}: D R_{\lambda}^{m, n} f \in \mathcal{S}(\xi, \phi)\right\} .
\end{aligned}
$$

In particular, we set

$$
\mathcal{S}_{\lambda}^{m, n}\left(\xi, \frac{1+A z}{1+B z}\right)=\mathcal{S}_{\lambda}^{m, n}(\xi, A, B), \quad-1<B<A \leq 1
$$

Next, we will investigate various inclusion relationships for the subclasses of analytic functions introduced above. Furthermore, we study the results of Faisal et al. [21], Darus and Faisal [3].

\section{Inclusion relationship associated with the operator $D R_{\lambda}^{m, n}$}

First, we start with the following lemmas which we need for our main results.

Lemma $2.1([22,23])$ Let $\varphi(\mu, v)$ be a complex function such that $\varphi: D \rightarrow \mathbb{C}, D \subseteq \mathbb{C} \times \mathbb{C}$, and let $\mu=\mu_{1}+i \mu_{2}, v=v_{1}+i v_{2}$. Suppose that $\varphi(\mu, v)$ satisfies the following conditions:

1. $\varphi(\mu, v)$ is continuous in $D$,

2. $(1,0) \in D$ and $\operatorname{Re} \varphi(1,0)>0$,

3. $\operatorname{Re} \varphi\left(i \mu_{2}, v_{1}\right) \leq 0$ for all $\left(i \mu_{2}, v_{1}\right) \in D$ such that $v_{1} \leq-\frac{1}{2}\left(1+\mu_{2}^{2}\right)$.

Let $h(z)=1+c_{1} z+c_{2} z^{2}+\cdots$ be analytic in $U$, such that $\left(h(z), z h^{\prime}(z)\right) \in D$ for all $z \in U$. If $\operatorname{Re}\left\{\varphi h(z), z h^{\prime}(z)\right\}>0, z \in U$, then $\operatorname{Re}\{h(z)\}>0$.

Lemma 2.2 ([24]) Let $\phi$ be convex univalent in $U$ with $\phi(0)=1$ and $\operatorname{Re}\{k \phi(z)+v\}>0$, $k, v \in \mathbb{C}$. If $p$ is analytic in $U$ with $p(0)=1$, then

$$
p(z)+\frac{z p^{\prime}(z)}{k p(z)+v} \prec \phi(z), \quad z \in U,
$$

implies $p(z) \prec \phi(z), z \in U$. 
Theorem 2.1 Let $f \in \mathcal{A}, 0 \leq \xi<1, m, n \in \mathbb{N}, \lambda>0$, then

$$
\mathcal{S}_{\lambda}^{m, n+1}(\xi) \subseteq \mathcal{S}_{\lambda}^{m, n}(\xi) \subseteq \mathcal{S}_{\lambda}^{m, n-1}(\xi)
$$

Proof Let $f \in \mathcal{S}_{\lambda}^{m, n+1}(\xi)$ and suppose that

$$
\frac{z\left(D R_{\lambda}^{m, n} f(z)\right)^{\prime}}{D R_{\lambda}^{m, n} f(z)}=\xi+(1-\xi) h(z) .
$$

Since from (1.3)

$$
(n+1) \frac{D R_{\lambda}^{m, n+1} f(z)}{D R_{\lambda}^{m, n} f(z)}=n+\xi+(1-\xi) h(z),
$$

we obtain

$$
\begin{aligned}
& (1-\xi) h^{\prime}(z)=(n+1)\left[\frac{\left(D R_{\lambda}^{m, n+1} f(z)\right)^{\prime}}{D R_{\lambda}^{m, n} f(z)}-\frac{D R_{\lambda}^{m, n+1} f(z)}{D R_{\lambda}^{m, n} f(z)} \cdot \frac{\left(D R_{\lambda}^{m, n} f(z)\right)^{\prime}}{D R_{\lambda}^{m, n} f(z)}\right], \\
& (1-\xi) z h^{\prime}(z)=(n+1) \frac{D R_{\lambda}^{m, n+1} f(z)}{D R_{\lambda}^{m, n} f(z)}\left[\frac{z\left(D R_{\lambda}^{m, n+1} f(z)\right)^{\prime}}{D R_{\lambda}^{m, n+1} f(z)}-\xi-(1-\xi) h(z)\right] \\
& \frac{(1-\xi) h^{\prime}(z) z}{n+\xi+(1-\xi) h(z)}=\frac{z\left(D R_{\lambda}^{m, n+1} f(z)\right)^{\prime}}{D R_{\lambda}^{m, n+1} f(z)}-\xi-(1-\xi) h(z) \\
& \frac{z\left(D R_{\lambda}^{m, n+1} f(z)\right)^{\prime}}{D R_{\lambda}^{m, n+1} f(z)}-\xi=(1-\xi) h(z)+\frac{(1-\xi) h^{\prime}(z) z}{n+\xi+(1-\xi) h(z)}
\end{aligned}
$$

Taking $h(z)=\mu=\mu_{1}+i \mu_{2}$ and $z h^{\prime}(z)=v=v_{1}+i v_{2}$, we define $\varphi(\mu, v)$ by

$$
\varphi(\mu, v)=(1-\xi) \mu+\frac{(1-\xi) v}{n+\xi+(1-\xi) \mu}
$$

and

$$
\begin{aligned}
& \operatorname{Re}\left\{\varphi\left(i \mu_{2}, \nu_{1}\right)\right\}=\frac{(1-\xi)(n+\xi) \nu_{1}}{(n+\xi)^{2}+(1-\xi)^{2} \mu_{2}^{2}} \\
& \operatorname{Re}\left\{\varphi\left(i \mu_{2}, \nu_{1}\right)\right\} \leq-\frac{(1-\xi)(n+\xi)\left(1+\mu_{2}^{2}\right)}{2\left[(n+\xi)^{2}+(1-\xi)^{2} \mu_{2}^{2}\right]}<0 .
\end{aligned}
$$

Clearly, $\varphi(\mu, v)$ satisfies the conditions of Lemma 2.1. Hence $\operatorname{Re}\{h(z)\}>0, z \in U$, implies $f \in \mathcal{S}_{\lambda}^{m, n}(\xi)$.

Remark 2.1 Using relation (1.2) and the same techniques as to prove the earlier results, we can obtain a new similar result.

Theorem 2.2 Let $f \in \mathcal{A}$ and $\phi \in \mathcal{R}$ with

$$
\operatorname{Re}\{\phi(z)\}<\frac{\xi-1+\frac{1}{\lambda}}{1-\xi}
$$


Then

$$
\mathcal{S}_{\lambda}^{m+1, n}(\xi, \phi) \subset \mathcal{S}_{\lambda}^{m, n}(\xi, \phi) \subset \mathcal{S}_{\lambda}^{m-1, n}(\xi, \phi)
$$

Proof Let $f(z) \in \mathcal{S}_{\lambda}^{m+1, n}(\xi, \phi)$ and set

$$
p(z)=\frac{1}{1-\xi}\left(\frac{z\left(D R_{\lambda}^{m, n} f(z)\right)^{\prime}}{D R_{\lambda}^{m, n} f(z)}-\xi\right)
$$

where $p$ is analytic in $U$ with $p(0)=1$.

By using (1.2) we have

$$
\frac{z\left(D R_{\lambda}^{m, n} f(z)\right)^{\prime}}{D R_{\lambda}^{m, n} f(z)}=\frac{1}{\lambda} \frac{D R_{\lambda}^{m+1, n} f(z)}{D R_{\lambda}^{m, n} f(z)}-\frac{1-\lambda}{\lambda} .
$$

Now, by using (2.2) we get

$$
\begin{aligned}
& p^{\prime}(z)=\frac{1}{1-\xi}\left(\frac{1}{\lambda} \frac{D R_{\lambda}^{m+1, n} f(z)}{D R_{\lambda}^{m, n} f(z)}-\frac{1-\lambda}{\lambda}-\xi\right), \\
& \frac{1}{\lambda} \frac{D R_{\lambda}^{m+1, n} f(z)}{D R_{\lambda}^{m, n} f(z)}=\xi+\frac{1-\lambda}{\lambda}+(1-\xi) p(z) .
\end{aligned}
$$

By using (2.2) and (2.3), we obtain

$$
\begin{aligned}
& z p^{\prime}(z)=\frac{1}{1-\xi} \cdot \frac{1}{\lambda}\left[\frac{z\left(D R_{\lambda}^{m+1, n} f(z)\right)^{\prime}}{D R_{\lambda}^{m, n} f(z)}-\frac{D R_{\lambda}^{m+1, n} f(z)}{D R_{\lambda}^{m, n} f(z)} \cdot \frac{z\left(D R_{\lambda}^{m, n} f(z)\right)^{\prime}}{D R_{\lambda}^{m, n} f(z)}\right], \\
& (1-\xi) z p^{\prime}(z)=\frac{1}{\lambda} \cdot \frac{D R_{\lambda}^{m+1, n} f(z)}{D R_{\lambda}^{m, n} f(z)}\left[\frac{z\left(D R_{\lambda}^{m+1, n} f(z)\right)^{\prime}}{D R_{\lambda}^{m+1, n} f(z)}-\frac{z\left(D R_{\lambda}^{m, n} f(z)\right)^{\prime}}{D R_{\lambda}^{m, n} f(z)}\right], \\
& (1-\xi) z p^{\prime}(z)=\left[\zeta-1+\frac{1}{\lambda}+(1-\xi) p(z)\right]\left[\frac{z\left(D R_{\lambda}^{m+1, n} f(z)\right)^{\prime}}{D R_{\lambda}^{m+1, n} f(z)}-(1-\xi) p(z)-\xi\right], \\
& \frac{(1-\xi) z p^{\prime}(z)}{(1-\xi) p(z)+\zeta-1+\frac{1}{\lambda}}=\frac{z\left(D R_{\lambda}^{m+1, n} f(z)\right)^{\prime}}{D R_{\lambda}^{m+1, n} f(z)}-\xi-(1-\xi) p(z) .
\end{aligned}
$$

Hence,

$$
\frac{1}{1-\xi}\left[\frac{z\left(D R_{\lambda}^{m+1, n} f(z)\right)^{\prime}}{D R_{\lambda}^{m+1, n} f(z)}-\xi\right]=p(z)+\frac{z p^{\prime}(z)}{(1-\zeta) p(z)+\zeta-1+\frac{1}{\lambda}} .
$$

Since $\operatorname{Re}\{\phi(z)\}<\frac{\xi-1+\frac{1}{\lambda}}{1-\xi}$ implies $\operatorname{Re}\left\{(1-\xi) p(z)+\xi-1+\frac{1}{\lambda}\right\}>0$, applying Lemma 2.2 to (2.4) we have that $f(z) \in \mathcal{S}_{\lambda}^{m, n}(\xi, \phi)$, as required.

Remark 2.2 By using relation (1.3) and the same techniques as to prove the earlier results, we can obtain a new similar result.

Corollary 2.3 Let $\frac{1+A}{1+B}<\frac{\xi-1+\frac{1}{\lambda}}{1-\xi}$ for $-1<B<A \leq 1$, then

$$
\mathcal{S}_{\lambda}^{m+1, n}(\xi, A, B) \subset \mathcal{S}_{\lambda}^{m, n}(\xi, A, B) \subset \mathcal{S}_{\lambda}^{m-1, n}(\xi, A, B) .
$$


Proof Taking $\phi(z)=\frac{1+A z}{1+B z},-1<B<A \leq 1$ in Theorem 2.2, we get the corollary.

\section{Integral-preserving properties}

In this section, we present several integral-preserving properties for the subclasses of analytic functions defined above. We recall the generalized Bernardi-Libera-Livington integral operator [25] defined by

$$
F_{c}[f(z)]=\frac{c+1}{z^{c}} \int_{0}^{z} t^{c-1} f(t) d t=z+\sum_{j=2}^{\infty} \frac{c+1}{j+c} a_{j} z^{c}, \quad f \in \mathcal{A}, c>-1,
$$

which satisfies the following equality:

$$
c D R_{\lambda}^{m, n} F_{c}[f(z)]+z\left[D R_{\lambda}^{m, n} F_{c}(f(z))\right]^{\prime}=(c+1) D R_{\lambda}^{m, n} f(z) .
$$

Theorem 3.1 Let $c>-1,0 \leq \xi<1$. Iff $\in \mathcal{S}_{\lambda}^{m, n}(\xi)$, then $F_{c} f \in \mathcal{S}_{\lambda}^{m, n}(\xi)$.

Proof Let $f \in \mathcal{S}_{\lambda}^{m, n}(\xi)$. By using (3.2), we get

$$
\frac{z\left[D R_{\lambda}^{m, n} F_{c}[f(z)]\right]^{\prime}}{D R_{\lambda}^{m, n} F_{c}[f(z)]}=(c+1) \frac{D R_{\lambda}^{m, n} f(z)}{D R_{\lambda}^{m, n} F_{c}[f(z)]}-c .
$$

Let

$$
\frac{z\left[D R_{\lambda}^{m, n} F_{c}[f(z)]\right]^{\prime}}{D R_{\lambda}^{m, n} F_{c}[f(z)]}=\xi+(1-\xi) h(z), \quad h(z)=1+c_{1} z+c_{2} z^{2}+\cdots .
$$

We obtain

$$
\frac{z\left[D R_{\lambda}^{m, n} f(z)\right]^{\prime}}{D R_{\lambda}^{m, n} f(z)}-\xi=(1-\xi) h(z)+\frac{(1-\xi) z h^{\prime}(z)}{\xi+(1-\xi) h(z)+c} .
$$

This implies

$$
\varphi(\mu, v)=(1-\xi) \mu+\frac{(1-\xi) v}{c+\xi+(1-\xi) \mu}
$$

(same as Theorem 2.1) and

$$
\begin{aligned}
& \operatorname{Re}\left\{\varphi\left(i \mu_{2}, v_{1}\right)\right\}=\frac{(1-\xi)(c+\xi) v_{1}}{(c+\xi)^{2}+(1-\xi)^{2} \mu_{2}^{2}}, \\
& \operatorname{Re}\left\{\varphi\left(i \mu_{2}, v_{1}\right)\right\} \leq-\frac{(1-\xi)(c+\xi)\left(1+\mu_{2}\right)^{2}}{2\left[(c+\xi)^{2}+(1-\xi)^{2} \mu_{2}^{2}\right]}<0 .
\end{aligned}
$$

After using Lemma 2.1 and Theorem 2.1, we have

$$
F_{c} f \in \mathcal{S}_{\lambda}^{m, n}(\xi) .
$$

Theorem 3.2 Let $c>-1$ and $\phi \in \mathcal{R}$ with

$$
\operatorname{Re}\{\phi(z)\}<\frac{c+\xi}{1-\xi} .
$$

Iff $\in \mathcal{S}_{\lambda}^{m, n}(\xi, \phi)$, then $F_{c} f \in \mathcal{S}_{\lambda}^{m, n}(\xi, \phi)$. 
Proof Let $f(z) \in \mathcal{S}_{\lambda}^{m, n}(\xi, \phi)$ and set

$$
p(z)=\frac{1}{1-\xi}\left(\frac{z\left[D R_{\lambda}^{m, n} F_{c}[f(z)]\right]^{\prime}}{D R_{\lambda}^{m, n} F_{c}[f(z)]}-\xi\right),
$$

where $p$ is analytic in $U$ with $p(0)=1$.

Using (3.2) and (3.3), we have

$$
(c+1) \frac{z\left[D R_{\lambda}^{m, n} f(z)\right]}{D R_{\lambda}^{m, n} F_{c}[f(z)]}=c+\xi+(1-\xi) p(z) .
$$

Then, using (3.2), (3.3) and (3.4), we obtain

$$
\frac{1}{1-\xi}\left(\frac{z\left[D R_{\lambda}^{m, n} f(z)\right]^{\prime}}{D R_{\lambda}^{m, n} f(z)}-\xi\right)=p(z)+\frac{z p^{\prime}(z)}{(1-\xi) p(z)+c+\xi} .
$$

Applying Lemma 2.2 to (3.5), we conclude that

$$
F_{c} f \in \mathcal{S}_{\lambda}^{m, n}(\xi, \phi) .
$$

\section{Competing interests}

The author declares that she has no competing interests.

\section{Author's contributions}

The author drafted the manuscript, read and approved the final manuscript.

\section{Acknowledgements}

The author thanks the referee for his/her valuable suggestions to improve the present article.

\section{Received: 16 March 2014 Accepted: 9 April 2014 Published: \#PUBLICATION_DATE}

\section{References}

1. Kumar, V, Shukla, SL: Certain integrals for classes of $p$-valent meromorphic functions. Bull. Aust. Math. Soc. 25, 85-97 (1982)

2. Miller, SS, Mocanu, PT: Differential Subordination. Dekker, New York (2000)

3. Darus, M, Faisal, I: Inclusion properties of certain subclasses of analytic functions. Rev. Notas Mat. 7(1)(305), 66-75 (2011)

4. Al-Oboudi, FM: On univalent functions defined by a generalized Sălăgean operator. Int. J. Math. Math. Sci. 27 1429-1436 (2004)

5. Sălăgean, GS: Subclasses of univalent functions. In: Complex Analysis - Fifth Romanian-Finnish Seminar. Lecture Notes in Math., vol. 1013, pp. 362-372. Springer, Berlin (1983)

6. Ruscheweyh, S: New criteria for univalent functions. Proc. Am. Math. Soc. 49, 109-115 (1975)

7. Andrei, L: Differential sandwich theorems using a generalized Sălăgean operator and Ruscheweyh operator. Didact. Math. (submitted)

8. Andrei, L: On some differential sandwich theorems using a generalized Sălăgean operator and Ruscheweyh operator. J. Comput. Anal. Appl. 18 (2015, to appear)

9. Andrei, L: Certain differential sandwich theorem using a generalized Sălăgean operator and Ruscheweyh operator. Adv. Appl. Math. Sci. (submitted)

10. Andrei, L: Differential subordinations, superordinations and sandwich theorems using a generalized Sălăgean operator and Ruscheweyh operator. Rev. Unión Mat. Argent. (submitted)

11. Alb Lupas, A: Certain differential subordinations using Sălăgean and Ruscheweyh operators. Acta Univ. Apulensis 29, 125-129(2012)

12. Alb Lupas, A: A note on differential subordinations using Sălăgean and Ruscheweyh operators. ROMAI J. 6(1), 1-4 (2010)

13. Alb Lupas, A: Certain differential superordinations using Sălăgean and Ruscheweyh operators. An. Univ. Oradea, Fasc. Mat. XVII(2), 209-216 (2010)

14. Alb Lupas, A: Certain differential subordinations using a generalized Sălăgean operator and Ruscheweyh operator J. Math. Appl. 33, 67-72 (2010)

15. Alb Lupas, A: Certain differential subordinations using a generalized Sălăgean operator and Ruscheweyh operator II. Fract. Calc. Appl. Anal. 13(4), 355-360 (2010)

16. Alb Lupas, A: Certain differential superordinations using a generalized Sălăgean and Ruscheweyh operators. Acta Univ. Apulensis 25, 31-40 (2011) 
17. Andrei, L: Differential subordination results using a generalized Sălăgean operator and Ruscheweyh operator. Acta Univ. Apulensis 37(2) (2014)

18. Andrei, L: Some differential subordination results using a generalized Sălăgean operator and Ruscheweyh operator. Jökull 64(4) (2014)

19. Andrei, L: Differential superordination results using a generalized Sălăgean operator and Ruscheweyh operator. An Univ. Oradea, Fasc. Mat. XXI(2) (2014, to appear)

20. Andrei, L: Some differential superordination results using a generalized Sălăgean operator and Ruscheweyh operator Stud. Univ. Babeş-Bolyai, Math. (to appear)

21. Faisal, I, Shareef, Z, Darus, M: On certain subclasses of analytic functions. Stud. Univ. Babeş-Bolyai, Math. 58(1), 9-14 (2013)

22. Miller, SS: Differential inequalities and Carathéordory function. Bull. Am. Math. Soc. 8, 79-81 (1975)

23. Miller, SS, Mocanu, PT: Second order differential inequalities in the complex plane. J. Math. Anal. Appl. 65, 289-305 (1978)

24. Eenigenberg, P, Miller, SS, Mocanu, PT, Reade, MO: On a Briot-Bouquet differential subordination. In: Genera Inequalities, vol. 3, pp. 339-348 (1983)

25. Bernardi, SD: Convex and starlike univalent functions. Trans. Am. Math. Soc. 135, 429-446 (1969)

\#DIGITAL_OBJECT_IDENTIFIER

Cite this article as: Andrei: Some properties of certain subclasses of analytic functions involving a differential operator. Advances in Difference Equations \#CITATION

\section{Submit your manuscript to a SpringerOpen ${ }^{\circ}$ journal and benefit from:}

- Convenient online submission

Rigorous peer review

- Immediate publication on acceptance

- Open access: articles freely available online

- High visibility within the field

- Retaining the copyright to your article 